contract. This includes the contractor's responsibilities in relation to managing associates and other members of staff and gives example models of ways to successfully divide contract value between practice associates. Complaint procedures and resolutions are then covered followed by a short chapter on clinical governance.

Finally, part four discusses the business considerations of running a successful NHS practice in an everchanging environment and how dental practitioners can prepare for and manage the changes which are to come.

In summary, a well structured and written book accumulating the knowledge of three practitioners whose vast experience within the NHS enables this book to be of relevance to all general dental practitioners including associates and practice owners.

\section{S. Williams}

\section{COLOR ATLAS OF DENTAL IMPLANT SURGERY, 3RD EDITION}

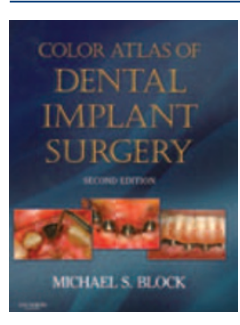

M. S. Block

UK: Elsevier

price $€ 139.00$; pp 480

ISBN 9781437708776

This is an organised and well structured book, providing the latest in dental implant surgery and associated grafting procedures. It acts as a surgical guide for the clinician covering every implant challenge and also contains an abundance of colour photographs which help towards clarifying concepts in implant dentistry. The topics addressed include: treatment planning, pre-surgical guidelines, detailed surgical techniques and postoperative follow-up. The book discusses extraction site protocols to reconstruct and preserve bone bulk and methods for grafting, to allow for implant placement at a later date.

Incorporated within the text are new methods for implant restoration, from single-tooth to multi-unit restorations along with attention to aesthetic considerations in relation to central incisors. The book also looks at immediate implant placement and the immediate loading of the edentulous mandible with provisional and final restorations, providing the patient with complete restoration of function within minutes of implant placement.

The book analyses the use of distraction osteogenesis for severely atrophic anterior maxillary sites and treatment of anterior maxillary atrophy with onlay grafts for fixed prosthetics, to restore the patient with severe maxillary defects and create the soft tissue needed for bone graft and later implant placement. The publication also discusses new techniques for bone morphogenetic protein for sinus augmentation, allowing implant placement, without the need for morbid autogenous bone harvesting or the use of allografts or xenografts.

A thoroughly enthralling and educational read, this is a must for every implant surgeon and advanced restorative clinician!

0. Morgan

\section{HARTY'S ENDODONTICS IN CLINICAL PRACTICE, 6TH EDITION}

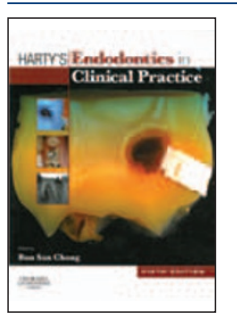

B. San Chong (ed)

UK: Elsevier

price $£ 53.99$; pp 312

ISBN 9780702031564

This book was originally written by Fred Harty, and has since evolved, maintaining its relevance to current practice and literature. This sixth edition has been dedicated to the late Tom Pitt Ford, Professor of Endodontology, who had edited previous texts. The introduction of this book of 'proven, current clinical endodontic practice' describes it as being intended for both 'dental practitioners seeking to update their knowledge' and for dental students in the UK as 'an essential undergraduate text'.

This 285 page paperback has 15 chapters. In contrast to other books, each chapter has been written by a different author with postgraduate qualifications and expertise on the individual topic and has been efficiently edited to include the salient points without labouring in excessive detail. Chapters follow a sound and structured layout including a contents page, background summary, introduction, learning outcomes and references.

The chapters are self explanatory and include an introduction/overview (outlining the scope of endodontics and recent developments); general and systemic aspects of endodontics; diagnosis; pulp anatomy; maintaining pulp vitality; basic instrumentation; preparation of the canal; intracanal medication; root canal obturation; surgical endodontics; primary teeth; traumatic injuries; periodontitis; endodontic problems; and restoration of the endodontically treated tooth.

The text greatly benefits from an abundance of labelled images throughout, including radiographs, coloured clinical photographs, diagrams, charts and tables. Unlike some related textbooks, the images are modern. Photographs and radiographs are of high quality and in many cases, clinical procedures have been illustrated in a stepwise series - such as access cavities; surgical endodontics (apicectomies and periodontal surgery); and restoration of the endodontically treated tooth (anterior and posterior intra-canal posts and extra-coronal restorations)

The number of revised editions of this book reflects continual advancements in this sub-speciality. This book is a simple one-step tool for keeping abreast of new techniques including rotary endodontics, electronic apex locators, and heated gutta-percha delivery systems. References have also been made to new UK guidelines of single-use instrumentation.

I used a previous edition of this text for my undergraduate learning, and I am impressed with how it has since advanced. Reviewing the current revised edition, I can see how it is highly relevant to both undergraduates and general dental practitioners.

K. Forde 